\title{
Classical equations of an electron from the special form of the Dirac equation
}

\author{
Miroslav Pardy \\ Department of Physical Electronics \\ and \\ Laboratory of Plasma physics \\ Masaryk University \\ Kotlářská 2, 61137 Brno, Czech Republic \\ e-mail:pamir@physics.muni.cz
}

October 30, 2021

\begin{abstract}
The equivalent system of equations corresponding to the Dirac equation is derived and the WKB approximation of this system is found. Similarly, the WKB approximation for the equivalent system of equation corresponding to the squared Dirac equation is found and it is proved that the Lorentz equation and the Bargmann-Michel-Telegdi iquations follow from the new Dirac-Pardy system. The new tensor equation with sigma matrix is derived for the verification by adequate laboratories.
\end{abstract}

Key words: Dirac equation, Lorentz equation, Bargmann-Michel-Telegdi equation, Dirac-Pardy equation.

\section{Introduction}

Bargmann, Michel and Telegdi (Berestetzkii, 1989;) derived so called BMT equation for motion of spin in the electromagnetic field, in the form

$$
\frac{d a_{\mu}}{d s}=\alpha F_{\mu \nu} a^{\nu}-\beta u_{\mu} F^{\nu \lambda} u_{\nu} a_{\lambda},
$$

where $a_{\mu}$ is so called axial vector describing the classical spin and constants $\alpha$ and $\beta$ were determined after the comparison of the postulated equations with the non-relativistic quantum mechanical limit. The result of such comparison is the final form of so called BMT equations: 


$$
\frac{d a_{\mu}}{d s}=2 \mu F_{\mu \nu} a^{\nu}-2 \mu^{\prime} u_{\mu} F^{\nu \lambda} u_{\nu} a_{\lambda}
$$

where $\mu$ is magnetic moment of electron following directly from the Dirac equation and $\mu^{\prime}$ is anomalous magnetic moment of electron which can be calculated as the radiative correction to the interaction of electron with electromagnetic field and follows from quantum electrodynamics. The BMT equation has more earlier origin. The first attempt to describe the spin motion in electromagnetic field using the special theory of relativity was performed by Thomas (1926). However, the basic ideas on the spin motion was established by Frenkel $(1926,1958)$. After appearing the Frenkel basic article, many authors published the articles concerning the spin motion (Ternov et al., 1980; Tomonaga, 1997). The mechanical model of spin was constructed by Uhlenbeck and Goudsmith (1926), or, in the very sophisticated form by Ohanian (1986) and other authors. However, we know at present time that spin of electron is its physical attribute which follows only from the Dirac equation. Also the Schrödinger Zitterbewegung of the Dirac electron as a point-like particle follows from the Dirac equation.

It was shown by Rafanelli and Schiller (1964), (Pardy, 1973) that the BMT equation can be derived from the classical limit, i.e. from the WKB solution of the Dirac equation with the anomalous magnetic moment. BMT equation is also the basic equation of the non-dissipative spintronics.

\section{The equivalent form of the Dirac equation}

Dirac equation is the quantum equation for particles with spin $1 / 2$. It has long history and let us submit some words on the spin.

Spin is an intrinsic form of angular momentum of elementary particles and of atomic nuclei. The electron spin angular momentum is inferred from experiments, such as the Stern-Gerlach experiment, in which silver atoms were observed to possess two possible discrete angular momenta despite having no orbital angular momentum.

Spin is described mathematically as a vector for some particles such as photons, and as spinors and bispinors for other particles such as electrons. Spinors and bispinors behave similarly to vectors: they have definite magnitudes and change under rotations; however, they use an unconventional "direction".

Now, let us approach the Dirac equation. We follow the five decades old original article (Pardy, 1973) and the monograph by well-known experts (Akhiezer et al., 1969; 1981):

$$
\varphi_{1}+\varphi_{2}+m c \psi=0
$$

where

$$
\varphi_{1}=\hbar \gamma_{\mu} \frac{\partial \psi}{\partial x_{\mu}} ; \quad \varphi_{2}=-i \frac{e}{c} A_{\mu} \gamma_{\mu} \psi
$$

Let us multiply eq. (3) from left by the spinors

$$
\bar{\psi} I, \quad \bar{\psi} \gamma_{\mu}, \quad \bar{\psi} \sigma_{\mu \nu}, \quad \bar{\psi} i \gamma_{5} \gamma_{\mu}, \quad \bar{\psi} \gamma_{5} ; \quad \gamma_{5}=\gamma_{1} \gamma_{2} \gamma_{3} \gamma_{4}
$$

where 


$$
\bar{\psi} \equiv \psi^{+} \gamma_{4} ; \quad \psi^{+} \equiv\left(\psi_{1}^{*}, \psi_{2}^{*}, \psi_{3}^{*}, \psi_{4}^{*}\right) ; \quad \sigma_{\mu \nu}=\frac{1}{2 i}\left(\gamma_{\mu} \gamma_{\nu}-\gamma_{\nu} \gamma_{\mu}\right)
$$

In this way we get the following system of equations:

$$
\begin{gathered}
\bar{\psi} \varphi_{1}+\bar{\psi} \varphi_{2}+m c \bar{\psi} \psi=0 \\
\bar{\psi} \gamma_{\mu} \varphi_{1}+\bar{\psi} \gamma_{\mu} \varphi_{2}+m c \bar{\psi} \gamma_{\mu} \psi=0 \\
\bar{\psi} \sigma_{\mu \nu} \varphi_{1}+\bar{\psi} \sigma_{\mu \nu} \varphi_{2}+m c \bar{\psi} \sigma_{\mu \nu} \psi=0 \\
\bar{\psi} i \gamma_{5} \gamma_{\mu} \varphi_{1}+\bar{\psi} i \gamma_{5} \gamma_{\mu} \varphi_{2}+m c \bar{\psi} i \gamma_{5} \gamma_{\mu} \psi=0 \\
\bar{\psi} \gamma_{5} \varphi_{1}+\bar{\psi} \gamma_{5} \varphi_{2}+m c \bar{\psi} \gamma_{5} \psi=0 .
\end{gathered}
$$

Now, let us consider that for matrices $\Gamma_{i}$

$$
\Gamma_{i} \equiv I, \quad \gamma_{\mu}, \quad \sigma_{\mu \nu}, \quad i \gamma_{5} \gamma_{\mu}, \quad \gamma_{5}
$$

The following identity holds good (Akhiezer et al., 1969; 1981):

$$
\frac{1}{4}\left(\sum_{i}\left(\Gamma_{i}\right)_{\alpha \omega}\left(\Gamma_{i}\right)_{\varrho \beta}\right)=\delta_{\alpha \beta} \delta_{\varrho \omega}
$$

Multiplying equation (13) by the spinor components $\varphi_{\beta}, \bar{\psi}_{\varrho}, \psi_{\omega}$, we get:

$$
\psi \cdot \bar{\psi} \varphi+\gamma_{\mu} \psi \cdot \bar{\psi} \gamma_{\mu} \varphi+\sigma_{\mu \nu} \psi \cdot \bar{\psi} \sigma_{\mu \nu} \varphi+i \gamma_{5} \gamma_{\mu} \psi \cdot \bar{\psi} i \gamma_{5} \gamma_{\mu} \varphi+\gamma_{5} \psi \cdot \bar{\psi} \gamma_{5} \varphi=4 \varphi(\psi \cdot \bar{\psi}) .
$$

In the equation (14) we do not use the the free spinor index $\alpha$. Puttin $\varphi=\psi$, we get the following identity from eq. (14):

$$
\psi \cdot \bar{\psi} \psi+\gamma_{\mu} \psi \cdot \bar{\psi} \gamma_{\mu} \psi+\sigma_{\mu \nu} \psi \cdot \bar{\psi} \sigma_{\mu \nu} \psi+i \gamma_{5} \gamma_{\mu} \psi \cdot \bar{\psi} i \gamma_{5} \gamma_{\mu} \psi+\gamma_{5} \psi \cdot \bar{\psi} \gamma_{5} \psi=4 \varphi(\psi \cdot \bar{\psi}) .
$$

Now, let us multiply eq. (7) by the spinor $\psi$, eq. (8) by the spinor $\gamma_{\mu} \psi$, eq. (9) by the spinor $\sigma_{\mu \nu} \psi$, eq. (10) by the spinor $i \gamma_{5} \gamma_{\mu} \psi$, eq. (11) by the spinor $\gamma_{5} \psi$ and let us sum up these resulting equations. With regard to eqs. (14) and (15) we get

$$
4(\bar{\psi} \psi)\left(\varphi_{1}+\varphi_{2}+m c \psi\right)=0,
$$

Assuming that $(\bar{\psi} \psi) \neq 0$, we have

$$
\varphi_{1}+\varphi_{2}+m c \psi=0 .
$$

The last equation is the Dirac equation again.

As shown, the Dirac equation follows unambiguously from the grand system (7-11) and at the same time this grand system $(7-11)$ can be derived from the Dirac equation. The system (7-11) and the Dirac equation are therefore equivalent to each other. In theoretical applications it is therefore possible to proceed both from the original Dirac equation and from the equivalent grand system (7-11). 


\section{WKB solution for the equivalent system to the Dirac equation}

This method is named after physicists Wentzel, Kramers, and Brillouin, who all developed it in 1926. In 1923, mathematician Harold Jeffreys had developed a general method of approximating solutions to linear, second-order differential equations, a class that includes the Schroedinger equation. Early quantum mechanics contained any number of combinations of their initials, including WBK, BWK, WKBJ, JWKB and BWKJ. The critical survey has been given by Dingle (1973).

Let us try to find the WKB solution of the system of eqs. (7-11). This asymptotic solution is the series in the small parameter $\hbar$ of the following form (Akhiezer et al., 1969; 1981):

$$
\psi=e^{\frac{i}{\hbar} S}\left(a_{0}+\hbar a_{1}+\hbar^{2} a_{2}+\ldots\right),
$$

where $S$ is the scalar real function and the coefficients $a_{0}, a_{1}, a_{2}, .$. are the four-component complex spinors.

It will suffice for our purposes, if we restrict series to the first term. For this reason we replace the function by the following one

$$
\psi_{W K B}=a_{0} e^{\frac{i}{\hbar} S}
$$

in the system $(7-11)$. If we annul the coefficients with $\hbar^{0}$ we get:

$$
\begin{gathered}
\left(\partial_{\mu} S-\frac{e}{c} A_{\mu}\right) \bar{a}_{0} \gamma_{\mu} a_{0}=i m c \bar{a}_{0} a_{0} \\
\left(\partial_{\mu} S-\frac{e}{c} A_{\mu}\right) \bar{a}_{0} \gamma_{\nu} \gamma_{\mu} a_{0}=i m c \bar{a}_{0} \gamma_{\nu} a_{0} \\
\left(\partial_{\mu} S-\frac{e}{c} A_{\mu}\right) \bar{a}_{0} \sigma_{\alpha \beta} \gamma_{\mu} a_{0}=i m c \bar{a}_{0} \sigma_{\alpha \beta} a_{0} \\
\left(\partial_{\mu} S-\frac{e}{c} A_{\mu}\right) \bar{a}_{0} \gamma_{5} \gamma_{\nu} \gamma_{\mu} a_{0}=i m c \bar{a}_{0} \gamma_{5} \gamma_{\nu} a_{0} \\
\left(\partial_{\mu} S-\frac{e}{c} A_{\mu}\right) \bar{a}_{0} \gamma_{5} \gamma_{\mu} a_{0}=i m c \bar{a}_{0} \gamma_{5} a_{0} .
\end{gathered}
$$

Let us put further $a_{0}=R \Phi$ where $R$ is the scalar function and $\Phi$ is the unit spinor. After some modification of eqs. (20-24), we get

$$
\begin{gathered}
\left(\partial_{\mu} S-\frac{e}{c} A_{\mu}\right) \bar{\Phi} \gamma_{\mu} \Phi=i m c \bar{\Phi} \Phi \\
\left(\partial_{\mu} S-\frac{e}{c} A_{\mu}\right) \bar{\Phi} \gamma_{\nu} \gamma_{\mu} \Phi=i m c \bar{\Phi} \gamma_{\nu} \Phi \\
\left(\partial_{\mu} S-\frac{e}{c} A_{\mu}\right) \bar{\Phi} \sigma_{\alpha \beta} \gamma_{\mu} \Phi=i m c \bar{\Phi} \sigma_{\alpha \beta} \Phi \\
\left(\partial_{\mu} S-\frac{e}{c} A_{\mu}\right) \bar{\Phi} \gamma_{5} \gamma_{\nu} \gamma_{\mu} \Phi=i m c \bar{\Phi} \gamma_{5} \gamma_{\nu} \Phi
\end{gathered}
$$




$$
\left(\partial_{\mu} S-\frac{e}{c} A_{\mu}\right) \bar{\Phi} \gamma_{5} \gamma_{\mu} \Phi=i m c \bar{\Phi} \gamma_{5} \Phi
$$

Now, we apply the similar procedure to the complex-conjugate of the original Dirac equation

$$
\bar{\varphi}_{1}+\bar{\varphi}_{2}+m c \bar{\psi}=0
$$

where we we substitute $\bar{\psi}$ by

$$
\bar{\psi}_{W K B}=e^{\frac{i}{\hbar} S} \bar{a}_{0}
$$

We obtain:

$$
\begin{gathered}
\left(\partial_{\mu} S-\frac{e}{c} A_{\mu}\right) \bar{\Phi} \gamma_{\mu} \Phi=i m c \bar{\Phi} \Phi \\
\left(\partial_{\mu} S-\frac{e}{c} A_{\mu}\right) \bar{\Phi} \gamma_{\mu} \gamma_{\nu} \Phi=i m c \bar{\Phi} \gamma_{\nu} \Phi \\
\left(\partial_{\mu} S-\frac{e}{c} A_{\mu}\right) \bar{\Phi} \gamma_{\mu} \sigma_{\alpha \beta} \Phi=i m c \bar{\Phi} \sigma_{\alpha \beta} \Phi \\
\left(\partial_{\mu} S-\frac{e}{c} A_{\mu}\right) \bar{\Phi} \gamma_{5} \gamma_{\nu} \gamma_{\mu} \Phi=i m c \bar{\Phi} \gamma_{\mu} \gamma_{5} \gamma_{\nu} \Phi \\
\left(\partial_{\mu} S-\frac{e}{c} A_{\mu}\right) \bar{\Phi} \gamma_{5} \gamma_{\mu} \Phi=i m c \bar{\Phi} \gamma_{5} \Phi .
\end{gathered}
$$

Let us try to interpret the quantity $i c \bar{\Phi} \gamma_{\mu} \Phi$. If we sum up eq (26a) and (26b) we get using relation

$$
\gamma_{\mu} \gamma_{\nu}+\gamma_{\nu} \gamma_{\mu}=\delta_{\mu \nu}
$$

the following equation:

$$
\left(\partial_{\mu} S-\frac{e}{c} A_{\mu}\right)=i m c \bar{\Phi} \gamma_{\mu} \Phi .
$$

However, according to classical mechanics, the following relations is valid

$$
\left(\partial_{\mu} S-\frac{e}{c} A_{\mu}\right)=m v_{\mu}
$$

where $v_{\mu}$ is the fou-vector of velocity. Therefore we put

$$
v_{\mu}=i c \bar{\Phi} \gamma_{\mu} \Phi
$$

By substituting $v_{\mu}$ into eqs. (25a) and (25b) respectively, we get that $\bar{\Phi} \Phi=1$, which is in agreement with the definition of the unit spinor $\phi$.

Subtracting eq. (28a) from (28b) we get $\bar{\Phi} \gamma_{5} \Phi=0$. Putting

$$
S_{\mu}=i \hbar \bar{\Phi} \gamma_{5} \gamma_{\mu} \Phi
$$


it follows from eqs. (29a) and (29b) respectively, that

$$
S_{\mu} v_{\mu}=0 .
$$

The quantity $S_{\mu}$ can be transformed as the axial vector, we can interpreted it as a spin.

Considering that the eight equations only, in the system (25a-25b) are independent, all information obtained by the WKB approximation of the Dirac equation is contained in the equation for $S_{\mu}$ and $v_{\mu}$. How to obtain the equations of motion for these quantities, will be shown in the following section.

\section{Dynamical equations for $v_{\mu}$ and $S_{\mu}$}

It would be possible to derive the dynamical equations for $v_{\mu}$ and $S_{\mu}$ directly from the system $(25 \mathrm{a}-29 \mathrm{a})$ and $(25 \mathrm{~b}-29 \mathrm{~b})$ but it is better to proceed from the squared Dirac equation. We obtain this equation by applying the operator

$$
\gamma_{\mu}\left(\hbar \frac{\partial}{\partial x_{\mu}}-i \frac{e}{c} A_{\mu}\right)-m c
$$

to the original Dirac equation. On performing the operation and after some modifications, we get:

$$
\left\{\pi_{\mu} \pi_{\mu}-\frac{i e \hbar}{2 c} F_{\mu \nu} \gamma_{\mu} \gamma_{\nu}-m^{2} c^{2}\right\} \psi=0
$$

where

$$
\pi_{\mu}=\left(\hbar \frac{\partial}{\partial x_{\mu}}-i \frac{e}{c} A_{\mu}\right)
$$

Now, we continue similarly as in the preceding section. We write down the system of equivalent equations for eq. (39) in which we substitute the function $\Phi$ by

$$
\psi_{W K B}=a_{0} e^{\frac{i}{\hbar} S} .
$$

We annul coefficients with $\hbar^{0}, \hbar^{1}$, put $a_{0}=R \Phi$ and finally we consider the fact that the continuity equation holds good.

$$
\partial_{\mu}\left(R^{2} v_{\mu}\right)=0 .
$$

If we further use the identity (Rafaneli et al., 1964)

$$
v_{\mu} \partial_{\mu} \equiv \frac{d}{d \tau}
$$

where $\tau$ is proper time, we get as a result of the whole procedure the following equations:

$$
\left\{\left(\partial_{\mu} S-\frac{e}{c} A_{\mu}\right)^{2}+m^{2} c^{2}\right\} \bar{\Phi} \Phi=0
$$




$$
\begin{gathered}
\left\{\left(\partial_{\mu} S-\frac{e}{c} A_{\mu}\right)^{2}+m^{2} c^{2}\right\} \bar{\Phi} \gamma_{\nu} \Phi=0 \\
\left\{\left(\partial_{\mu} S-\frac{e}{c} A_{\mu}\right)^{2}+m^{2} c^{2}\right\} \Phi \sigma_{\alpha \beta} \gamma_{\nu} \Phi=0 \\
\left\{\left(\partial_{\mu} S-\frac{e}{c} A_{\mu}\right)^{2}+m^{2} c^{2}\right\} \bar{\Phi} \gamma_{5} \gamma_{\nu} \Phi=0 \\
\left\{\left(\partial_{\mu} S-\frac{e}{c} A_{\mu}\right)^{2}+m^{2} c^{2}\right\} \bar{\Phi} \gamma_{5} \Phi=0 \\
\bar{\Phi} \frac{d \Phi}{d \tau}=-\frac{e}{4 m c} \bar{\Phi} \gamma_{\varrho} \gamma_{\omega} \Phi F_{\varrho \omega} \\
\bar{\Phi} \gamma_{\mu} \frac{d \Phi}{d \tau}=-\frac{e}{4 m c} \bar{\Phi} \gamma_{\mu} \gamma_{\varrho} \gamma_{\omega} \Phi F_{\varrho \omega} \\
\bar{\Phi} \sigma_{\alpha \beta} \frac{d \Phi}{d \tau}=-\frac{e}{4 m c} \bar{\Phi} \sigma_{\alpha \beta} \gamma_{\varrho} \gamma_{\omega} \Phi F_{\varrho \omega} \\
\bar{\Phi} \gamma_{5} \gamma_{\mu} \frac{d \Phi}{d \tau}=-\frac{e}{4 m c} \bar{\Phi} \gamma_{5} \gamma_{\mu} \gamma_{\varrho} \gamma_{\omega} \Phi F_{\varrho \omega} \\
\bar{\Phi} \gamma_{5} \frac{d \Phi}{d \tau}=-\frac{e}{4 m c} \bar{\Phi} \gamma_{5} \gamma_{\varrho} \gamma_{\omega} \Phi F_{\varrho \omega} .
\end{gathered}
$$

and

$$
\begin{aligned}
\frac{d \bar{\Phi}}{d \tau} \Phi & =\frac{e}{4 m c} \bar{\Phi} \gamma_{\varrho} \gamma_{\omega} \Phi F_{\varrho \omega} \\
\frac{d \bar{\Phi}}{d \tau} \gamma_{\mu} \Phi & =\frac{e}{4 m c} \bar{\Phi} \gamma_{\varrho} \gamma_{\omega} \gamma_{\mu} \Phi F_{\varrho \omega} \\
\frac{d \bar{\Phi}}{d \tau} \sigma_{\alpha \beta} \Phi & =\frac{e}{4 m c} \bar{\Phi} \gamma_{\varrho} \gamma_{\omega} \sigma_{\alpha \beta} \Phi F_{\varrho \omega} \\
\frac{d \bar{\Phi}}{d \tau} \gamma_{5} \gamma_{\mu} \Phi & =\frac{e}{4 m c} \bar{\Phi} \gamma_{\varrho} \gamma_{\omega} \gamma_{5} \gamma_{\mu} \Phi F_{\varrho \omega} \\
\frac{d \bar{\Phi}}{d \tau} \gamma_{5} \Phi & =\frac{e}{4 m c} \bar{\Phi} \gamma_{\varrho} \gamma_{\omega} \gamma_{5} \Phi F_{\varrho \omega} .
\end{aligned}
$$

It is easy to prove that by summing eqs. (49a) and (49b), (50a) and (50b), (51a) and (51b), (52a) and (52b), (53a) and (53b) we get

$$
\frac{d}{d \tau} \Phi \Phi=0
$$




$$
\begin{gathered}
-\frac{e}{c} F_{\mu \nu} v_{\nu}=m \frac{d v_{\mu}}{d \tau} \\
\frac{e}{4 m c}\left\{\bar{\Phi}\left(\gamma_{\varrho} \gamma_{\omega} \sigma_{\alpha \beta}-\sigma_{\alpha \beta} \gamma_{\varrho} \gamma_{\omega}\right) \Phi\right\} F_{\varrho \omega}=\frac{d}{d \tau} \bar{\Phi} \sigma_{\alpha \beta} \Phi \\
-\frac{e}{m c} F_{\mu \nu} S_{\nu}=\frac{d S_{\mu}}{d \tau} \\
\frac{d}{d \tau} \bar{\Phi} \gamma_{5} \Phi=0 .
\end{gathered}
$$

Equation (55) is the Lorentz equation of motion of charged particle in electromagnetic field.

Equation (57) is the Bargmann-Michel-Telegdi equation for the spin motion of the spin $1 / 2$ particle with a zero anomalous magnetic moment.

Equation (56) is the original equation following from the WKB approximation of the Dirac-Pardy equation, which was not derived by Schiller or Rafanelli, or others. The experimental verification of this equation is equivalent to the discovery following from the Dirac-Pardy equation.

\section{Discussion}

We have derived the equivalent system of equations corresponding to the Dirac equation and the WKB approximation of this system has been found. Similarly, the WKB approximation for the equivalent system of equation corresponding to the squared Dirac equation was found and it was proved that the Lorentz equation and the BargmannMichel-Telegdi iquations follow from the new Dirac-Pardy system. The new tensor equation with sigma matrix was derived and it is not excluded that the verification of this author original equation will be performed by such adequate laboratories as CERN. Equation (56) is the original tensor equation following from the Dirac-Pardy system and it was not derived by Schiller or Rafanelli, or, by the other theorists. This author equation was not considered in physics for five decades (Pardy, 1973). We have analogy in the history of science, where the Mendel, Heaviside, Planck, Moessbauer, Sommerfeld, and so on, ideas were ignored for some decades. Nevertheless, the experimental verification of the author ideas will be, no doubt, interesting and the grand Dirac-Pardy system will be considered as relevant.

\section{References.}

Akhiezer, A. I., and Berestetskii, V. B. (1981). Kvantovaya elektrodinamika. 3rd ed. [Quantum Electrodynamics] (Moscow: Nauka). (in Russian).

Akhiezer, A. N. Berestetzkii, V. B. (1969). Quantum electrodynamics, 3rd ed., (Moscow, Nauka). (in Russian).

Berestetzkii, V. B., Lifshitz, E. M. and Pitaevskii L. P., (1989). Quantum electrodynamics, 3rd ed., (Moscow, Nauka). (in Russian). 
Dingle, R. B. Asymptotic Expansions: Their Derivation and Interpretation (Academic Press, 1973).

Frenkel, J. I., (1926). Die Elektrodynamik der rotierenden Elektronen, Zs. Physik 37, 243.

Frenkel, J. I., (1958). Collective scientific works, II., Scientific articles, AN SSSR, (in Russian).

Landau, L. D. and Lifshitz, E. M., (1988). The classical theory of fields, 7th ed., (Moscow, Nauka), (in Russian).

Ohanian, H. C., (1986). What is spin?, Am. J. Phys. 54(6), 500.

Pardy, M., (1973). Classical motion of spin 1/2 particles with zero anomalous magnetic moment, Acta Phys. Slovaca 23, No. 1, 5.

Rafanelli, K, and Schiller, R., (1964). Classical motion of spin-1/2 particles, Phys. Rev. 135, No. 1 B, B279.

Ternov, I. M., (1980). On the contemporary interpretation of the classical theory of the J. I. Frenkel spin, Uspekhi fizicheskih nauk, 132, 345. (in Russian).

Thomas, L. H., (1926). The motion of spinning electron, Nature, 117, 514.

Tomonaga, S.-I., (1997). The story of spin, (The university of Chicago press, Ltd., London).

Uhlenbeck, G. E. and Goudsmit, S. A., (1926). Spinning electrons and the structure of spectra, Nature 117, 264. 